\title{
Effect of Walnut Consumption on Serum Lipid Profiles, High-Sensitivity C-Reactive Protein and Nitric Oxide in Patients With Coronary Artery Disease
}

\author{
Giti Kalantarian $^{1, *}$; Mohsen Rezaei $^{1}$; Shahram Homayonfar ${ }^{2}$; Marzieh Gharibi $^{3}$ \\ ${ }_{1}^{1}$ Department of Biochemistry, Faculty of Medicine, Hamadan University of Medical Sciences, Hamadan, IR Iran \\ ${ }^{2}$ Department of Health, Hamadan University of Medical Sciences, Hamadan, IR Iran \\ ${ }^{3}$ Department of Biochemistry, Bushehr University of Medical Sciences, Bushehr, IR Iran \\ ${ }^{*}$ Corresponding author: Giti Kalantarian, Department of Biochemistry, Hamadan University of Medical Sciences, Hamadan, IR Iran. Tel: +98-9163136471, Fax: +98-8118276299, E-mail: \\ giti_kalantarian@yahoo.com
}

Received: January 21, 2015; Accepted: January 24, 2015

\begin{abstract}
Background: Coronary artery disease (CAD) is one of the major causes of death worldwide. There is a direct relationship between increased levels of blood cholesterol, triglycerides (TG), low density lipoproteins (LDL), high density lipoproteins (HDL), nitric oxide (NO), and high sensitivity C-reactive protein (hs-CRP) and the CAD. Walnut may reduce these factors and consequently decrease the risk of CAD. Objectives: The aim of this study was to evaluate the effect of walnut consumption on TG, LDL, cholesterol, HDL, NO, and hs-CRP in patients with CAD and healthy people.

Patients and Methods: In this randomized crossover clinical trial, 70 patients with CAD were divided into two groups: case and control groups. The patients were given $40 \mathrm{~g}$ walnuts daily for 4 weeks. After 4 weeks, the TG, LDL, cholesterol, HDL, NO and hs-CRP levels were measured. The experiment was also carried out on healthy people (3 groups) with normal range of lipid. The LDL, HDL, cholesterol, and hs-CRP levels were measured using commercially available kits. The nitric oxide level was measured using the Griess reaction method.

Results: The cholesterol and LDL levels decreased significantly from 202.43 to 187.46 and 123.80 to $108.63 \mathrm{mmol} / \mathrm{L}$ (7.9\% and $13.9 \%$ ), respectively in healthy subjects after the treatment $(\mathrm{P}<0.01)$. In the experimental group, there was no significant difference in cholesterol $(\mathrm{P}=0.110)$ and LDL levels $(\mathrm{P}=0.176)$ before and after the treatment. Moreover, no significant difference was observed in other parameters between the two groups.

Conclusions: The walnut consumption did not affect cholesterol levels in patients with CAD; however, it might be administered as an agent for reducing the cholesterol, which is one of the risk factors associated with CAD.
\end{abstract}

Keywords: Walnut; Cholesterol; Triglycerides; Coronary Artery Disease

\section{Background}

Coronary artery disease (CAD) is one of the major causes of death and disability worldwide $(1,2)$. Ischemic heart diseases account for half of the total deaths in developed countries (3). Coronary heart disease (CHD) is one of the leading causes of disease burden in developing countries (4). According to the Iran's health ministry, $46 \%$ of the total death rate is due to cardiovascular diseases in Iran (5). Studies on arteriosclerosis indicate that fatty streaks represent as the primary indicator of arteriosclerosis, which results from focal increasing of lipoproteins in intima layer of the artery. These lipoproteins can accumulate by binding to the extracellular components of the arterial intima and hence enhance the residence time of the lipid rich particles within the arterial wall (6). Epidemiological and experimental studies showed that increasing in blood cholesterol is considered as the most important risk factor associated with CHD in which one percent increase in blood cholesterol would enhance a two-percent increase in the CHD incidence (7). Variation in a diet-fat quality of people with hyperlipidemia is of special importance and walnuts are among the foods recommended to reduce blood lipid. Walnut consists of poly-unsaturated fatty acid (PUFA), $47 \%$ of total weight, mainly linoleic acid (LA) and alpha-linolenic acid (ALA), 9\%, and it is free of cholesterol and trans fatty acids and is unique due to having $\operatorname{ALA}(8,9)$. Walnuts contain the amino acid L-arginine, which is used for the nitric oxide (NO) formation. The NO is a substance, which is mandatory to keep heart flexibility. In addition, walnuts contain phytosterols, which are beneficial for heart (10). Due to having poly-phenolic substances which are regarded among strong vitamin $\mathrm{E}$ antioxidant ingredients, walnuts decrease LDL and prevent from arteriosclerosis (11). Walnut is a rich source of fiber in which there is almost $9.7 \mathrm{~g}$ of fiber per $100 \mathrm{~g}$ of it. It was shown that fiber-rich foods can reduce cholesterol levels and prevent heart diseases and strokes (12). There are controversial reports regarding the effect of walnut consumption on blood lipid. Some reports showed an

Copyright (C) 2015, Ahvaz Jundishapur University of Medical Sciences. This is an open-access article distributed under the terms of the Creative Commons Attribution-NonCommercial 4.0 International License (http://creativecommons.org/licenses/by-nc/4.0/) which permits copy and redistribute the material just in noncommercial usages, provided the original work is properly cited. 
increase in high-density lipoprotein cholesterol (HDLC) while others demonstrated a decrease in HDL-C levels. However, in majority of the studies it was shown that walnut did not have any effect on HDL-C levels (13, 14). In addition, few studies pointed to walnut effect in decreasing the blood triglyceride levels (15). However, there is no data to verify these effects (16). Based on the previous reports, walnut consumption showed a reduction in low-density lipoprotein cholesterol (LDL-C) and total cholesterol $(\mathrm{TC})(16,17)$. While previous studies indicated that LA and ALA cause a decrease in the Creactive protein (hs-CRP) level, there are few reports on these effects $(17,18)$. Walnuts contain a very high level of arginine, a substrate for NO synthase enzyme to be turned into cytrolin and NO, and hence it is expected that walnut consumption would cause an increase in NO level. Walnut is a plant-based essential omega-3 fatty acid that has been shown to elicit anti-inflammatory and antiatherogenic effects (9).

\section{Objectives}

This study aimed to examine the effect of walnut consumption on serum lipids as well as serum hs-CRP and NO levels in patients with CAD, who had medical files in Hamadan Ekbatan Hospital (Iran), and healthy people.

\section{Patients and Methods}

A crossover clinical trial was conducted on 70 patients with CAD (60 males and 10 females, age range of 40-85 years) having medical files in Hamadan Ekbatan Hospital. To calculate the sample size, we used the mean and standard deviation of the results of other similar studies conducted in other countries previously. Seventy people were selected and the samples were calculated at the $95 \%$ confidence interval and. The patients were allocated into two-equal groups, case and control, to perform a crossover study in which both groups were similar and only varied in their diets. To enroll in the study, the participants should not have metabolic disorders such as diabetes and goiter; they should not smoke and should not be allergic to walnut and should not be taking cholesterol lowering drugs or supplements or nitrate compounds. However, practically we could not apply the last standard by patients. The protocol and consent form were approved by the Ethics Committee of Hamadan University of Medical Sciences (K/1/14/31).The case group received normal diet plus walnuts and the control group received only normal diet. The walnut was taken as three servings per day. Participants consumed three servings daily for one month and the consumption was controlled by the nurses. Three servings consisted of 13.3 grams of walnuts with each meal and a total consumption of 40 grams daily. The diet continued for 4 weeks. After 4 weeks, blood samples were taken from the participants in both case and control groups. Since according to some studies, plasma lipids and lipoproteins would be stabilized in less than 4 weeks(e.g. 2-3 weeks) and in the other studies there is no washout period $(8,19)$, in this study we had a 2-week washout period to be sure that the level of lipids and lipoproteins in plasma is stabilized (20); the same diet regimen was conducted in two groups for another 4 weeks, while the case and control groups were displaced. In this round, the control group was regarded as the case with a normal diet and daily walnut consumption and vice versa. The blood samples were taken from the participants in both groups (case and control) at the end of 4 weeks. Blood samples were collected in the morning after an overnight fast and were allowed to clot at room temperature for 1 hour. Serum levels were separated by centrifugation and kept at $-80^{\circ} \mathrm{C}$ until the analysis. The second part of the study was conducted to examine the effect of walnut consumption on healthy people. The healthy participates were selected in a way to have no significant difference in age and gender with the groups of patients with CAD. Similarly, healthy participants consumed three servings daily for one month. Three servings consisted of 13.3 grams of walnuts with each meal and a total consumption of 40 grams daily. To make sure that all the people in healthy group followed the diet regularly, every week we control the walnut consumption by the phone to make sure about the daily consumption of walnut. After 4 weeks, blood samples were collected in the morning after an overnight fast and were allowed to clot at room temperature for 1 hour. Serum were separated by centrifugation and kept at $-80^{\circ} \mathrm{C}$ until the analysis. Triglyceride, cholesterol, LDL-C, HDL and TC were measured using commercially available kits (Pars Azmoon, Iran). The level of NO was measured using the Griess reaction method. Moreover, the hs-CRP level was measured by commercial kits (Pars Azmoon, Iran) (21, 22). T-test was used to compare changes in outcome variables in response to the dietary treatment. Analyses were performed with SPSS software version 13.0.

\section{Results}

The mean age of patients with CAD was $57.07 \pm 11.56$ years, which showed no significant difference compared with the mean age of healthy participants $(53.73 \pm 6.49$ years). No significant difference was observed in the mean levels of cholesterol, LDL, HDL in the CAD patients with or without walnut consumption (Table 1). There was a significant difference in the mean levels of LDL and cholesterol before and after walnut consumption in healthy people group $(\mathrm{P}<0.01)$. As indicated in Table 2 , LDL and cholesterol levels were lower after daily walnut consumption in healthy participants as compared with those who didn't get walnut. Results showed no significant difference in these parameters after taking daily walnut inpatients with CAD. The addition of walnut in diet resulted in reducing total cholesterol and LDL-C levels; however, it did not affect the TG, HDL-C, hs-CRP and NO levels in healthy people. 
Kalantarian G et al.

Table 1. Comparison of the Levels of Parameters in With or Without Taking Walnut Groups in Patients With CAD, Coronary Artery Disease $^{\text {a }}$

\begin{tabular}{|c|c|c|c|c|}
\hline Time & Variation Domain & Average & Standard Deviation & PValue \\
\hline Cholesterol, mmol/L & & & & 0.110 \\
\hline Without taking & $103-351 / 00$ & $159 / 59$ & 43.56 & \\
\hline Taking & $115-470 / 00$ & 203.43 & 50.66 & \\
\hline Triglycerides, mmol/L & & & & 0.437 \\
\hline Without taking & $46-628 / 00$ & 201.86 & 111.72 & \\
\hline Taking & $51-796 / 00$ & 209.07 & 124.20 & \\
\hline HDL, mmol/L & & & & 0.085 \\
\hline Without taking & $14-59 / 00$ & 35.30 & 9.76 & \\
\hline Taking & $17-58.00$ & 36.70 & 9.86 & \\
\hline LDL, $\mathrm{mmol} / \mathrm{L}$ & & & & 0.176 \\
\hline Without taking & $51-187.00$ & 119.47 & 34.52 & \\
\hline Taking & $51.4-301.80$ & 125.30 & 39.23 & \\
\hline $\begin{array}{l}\text { Cholesterol/HDL, } \\
\text { mmol/L }\end{array}$ & & & & 0.929 \\
\hline Without taking & $2.6-14.00$ & 5.84 & 1.84 & \\
\hline Taking & $2.8-12.80$ & 5.81 & 1.98 & \\
\hline hs-CRP, nmol/L & & & & 0.154 \\
\hline Without taking & $38-18.00$ & 4.37 & 3.55 & \\
\hline Taking & $16-20.00$ & 5.32 & 4.82 & \\
\hline NO, nmol/L & & & & 0.358 \\
\hline Without taking & $07-3.81$ & 1.33 & 89 & \\
\hline Taking & $05-8.90$ & 1.54 & 1.46 & \\
\hline
\end{tabular}

a Abbreviations: HDL, High Density Lipoprotein; LDL, Low Density Lipoprotein; hs-CRP, High-Sensitivey C-Reactive Protein; NO, Nitric Oxide.

\begin{tabular}{|c|c|c|c|c|}
\hline Time & Variation Domain & Average & Standard Deviation & P Value \\
\hline \multicolumn{5}{|c|}{ Cholesterol, mmol/L } \\
\hline Before & $134-287.00$ & 202.43 & 38.73 & 0.002 \\
\hline After & $127-262.00$ & 187.46 & 36.76 & \\
\hline \multicolumn{5}{|c|}{ Triglycerides, mmol/L } \\
\hline Before & $70-331.00$ & 143.16 & 59.73 & 0.735 \\
\hline After & $65-302.00$ & 145.73 & 70.04 & \\
\hline \multicolumn{5}{|c|}{ HDL, mmol/L } \\
\hline Before & $36-69.00$ & 50.96 & 8.79 & 0.099 \\
\hline After & $40-61.00$ & 49.33 & 6.33 & \\
\hline \multicolumn{5}{|c|}{ LDL, $\mathrm{mmol} / \mathrm{L}$} \\
\hline Before & $70-199.00$ & 123.80 & 35.58 & 0.001 \\
\hline After & $61-200.00$ & 108.63 & 34.45 & \\
\hline \multicolumn{5}{|c|}{ Cholesterol/HDL, mmol/L } \\
\hline Before & $2.6-6.20$ & 3.98 & 0.93 & 0.617 \\
\hline After & $2.5-6.30$ & 3.93 & 1.07 & \\
\hline \multicolumn{5}{|c|}{ hs-CRP, nmol/L } \\
\hline Before & $0.2-10.00$ & 3.04 & 2.88 & 0.855 \\
\hline After & $0.4-8.00$ & 3.10 & 2.16 & \\
\hline \multicolumn{5}{|c|}{ NO, nmol/L } \\
\hline Before & $0.8-2.30$ & 1.51 & 0.37 & 0.118 \\
\hline After & $1.04-2.30$ & 1.65 & 0.32 & \\
\hline
\end{tabular}

\footnotetext{
a Abbreviations: HDL, High Density Lipoprotein; LDL, Low Density Lipoprotein; hs-CRP, High-Sensitivey C-Reactive Protein; NO, Nitric Oxide.
} 


\section{Discussion}

Decreasing the cholesterol and LDL-C levels in this study are in accordance with previous studies that showed taking walnuts in diet caused a reduction in total cholesterol and LDL levels $(7,8,13)$. Previous studies $(7,8,23)$ showed the hypocholesterolemic effect of walnut in males and females with hypocholesterolemia after daily taking of 56,50 and 48 grams of walnuts, respectively $(7,8,13)$. Other studies showed walnut hypocholestrolemic effects in healthy males and females while taking 58, 84 and 68 grams of daily walnut, respectively (14-16).Alpha-linolenic acid in walnut reduces LDL-C concentration through increasing the LDL-C receptor activity, prevention of VLDL to LDL-C conversion, decreasing in VLDL synthesis, and by increasing in LDL-C particle tendency to attach LDL-C receptors in hepatic $\mathrm{G} 2$ cells due to transformation of LDLC particle complemented with $\operatorname{ALA}(24,25)$. Sabate et al. reported that serum TG level reduced in healthy people after taking 84 grams of daily walnuts (15) while our data were in agreement with other studies that walnuts exert no effect in reducing blood triglyceride $(7,8,13)$. The effect of long-chain omega-3 fatty acid in reducing blood triglyceride in patients with hypertriglyceridemia has been reported to be intermediate $(26,27)$. It was hypothesized that the walnut ALA as a precursor for Eicosapantenoicacid (EPA) and Docosahexaenoicacid (DHA) exert similar effect as long-chain omega-3 PUFA fatty acid in reducing blood TG $(28,29)$. However, in this study walnuts had no effect in dropping blood TG which might be due to a competition between LA and ALA for denaturizing the enzyme. The LA/ALA ratio of around 4 is suitable for conversion of ALA into a long change metabolite $(30,31)$. In the present study, this ratio was possibly more than 4 , which consequently reduced the potential of the conversion of the ALA to the long-chain metabolite. The increase in EPA occurs with taking oils rich in ALA with low LA/ALA ratio of less than 3 which could not be simply reached by introducing of walnut to the daily diet $(13,32)$. Therefore, ALA and PUFA fatty acid (marine omega-3 type) have unique physiologic characteristics (33). According to the conducted research, marine sources of omega-3 fatty acid such as fish oil have higher hypotriglyceridemic effects compared to ALA, particularly in patients with hypertriglyceridemia (34). Another affecting reason for the lack of walnut effect on triglyceride level is the high ratio of PUFA to mono-unsaturated fatty acid (MUFA) in walnut. Some studies indicated that MUFA fatty acids have higher hypotriglyceride characteristics than PUFA (19). Based on the results of the present study, the triglyceride level was not reduced after 40 grams of daily walnut consumption, which was in line with other studies that walnut exert no effect on blood triglyceride levels $(7,8,13)$. In addition, no significant difference was observed in HDL-C level which was in line with most of the previous studies mentioned the lack of walnut effect on HDL-C level $(8,23)$. According to Avedrine (1991), HDL-C level increased after taking walnuts on daily diet (35). Clinical studies showed that taking high-lipid diet increases HDL-C levels through increasing the apolipoprotein $\mathrm{Al}$ in transportation or reducing its catabolic speed, conversely low fat diet drops the HDL-C levels through lowering lipoprotein lipase activity which hence resulted in a reduction in VLDL hydrolysis and HDL-C production $(36,37)$. In addition, lecitine cholesterol aciltransferase (LCAT) facilitates taking and joining cholesterol by HDL-C. Generally, PUFA fatty acids are weak substrates for Phosphatidylcholine-esterol O-acyltransferase (38). This might justify a reduction in HDL-C levels after walnut consumption in this study. No significant difference was observed in TC/(HDL-C) ratio. Walnuts consist of botanical proteins, fibers, vitamin E, folic acid, niacine, pyridoxine, and minerals such as magnesium, zinc, cooper, and potassium. Walnuts are also a source of active biological compounds, such as ellagicacid, flavonoids and phenelic compounds $(39,40)$. Botanical sterols in walnut may play a role in its hypolipidemic characteristics. Botanical sterols exist in nuts can drop the cholesterol level by blocking its absorbance $(41,42)$. Walnuts are rich in arginine (43) and low lysine to arginine ratio plays a role in serum cholesterol level (40). Saturation of LDL$\mathrm{C}$ particles with PUFA fatty acids in walnut does not alter their resistance to oxidative stress. Walnut due to having antioxidant properties, alpha tocopherols and other phytochemicals and polyphenolics inhibit LDL-C oxidation $(44,45)$. In conclusion, the present study showed that introducing walnuts in healthy people diet could significantly reduce TC and LDL-C levels in people having normal cholesterol levels, but such variations was not observed in patients with CAD. This might be possibly due to medicine interference or it is likely that walnut effects would be achieved in higher dosage of consumption in people with CAD. No variations in NO and hs-CRP levels were observed in both healthy participants and patients with CAD. As indicated before, increasing in blood cholesterol is considered as the most important risk factor associated with CHD. Although walnut consumption did not affect cholesterol levels in patients with $\mathrm{CAD}$, the daily walnut consumption would lower blood cholesterol and might be administrated as an agent for reducing the cholesterol level, which is one of the risk factors in CAD. Further investigation should be conducted to evaluate whether the increase in daily walnut consumption and administration dosage might cause a reduction in cholesterol levels in patients with CAD. Although the walnut consumption did not affect cholesterol levels in patients with CAD in this study, it may be due to the low-dose of walnut and thus by increasing the amount of walnut consumption this factor may be decreased (46); therefore, by increasing the intervention period, the risk factors may be decreased too (47). Although in this study, the walnut daily consumption can decrease the blood cholesterol and LDL levels in healthy people, the effects of walnut on the other factors need to be further investigated. 


\section{Acknowledgements}

The authors would like to thank the Hamadan University of Medical Sciences, Biochemistry Department for the financial support. We are also grateful to Dr. Rezai, Dr. Mahjoob, Dr. Goodarzi, Dr. Homayounfar and the staff of the Hamadan Ekbatan Hospital for their assistance and support.

\section{References}

1. Nussbaumerová B, Rosolová H, Mayer O, Filipovský J, Cífková R, Bruthans J. Residual cardiovascular risk in patients with stable coronary heart disease over the last 16 years (Czech part of the EUROASPIRE I-IV surveys). Cor Vasa. 2014;56(2):e98-e104.

2. Farkouh ME, Boden WE, Bittner V, Muratov V, Hartigan P, Ogdie $\mathrm{M}$, et al. Risk factor control for coronary artery disease secondary prevention in large randomized trials. J Am Coll Cardiol. 2013;61(15):1607-15.

3. Dawar R, Gurtoo A, Singh R. Apolipoprotein A1 gene polymorphism (G-75A and $\mathrm{C}+83 \mathrm{~T})$ in patients with myocardial infarction: a pilot study in a north Indian population. Am J Clin Pathol. 2010;134(2):249-55.

4. Gaziano TA, Bitton A, Anand S, Abrahams-Gessel S, Murphy A Growing epidemic of coronary heart disease in low- and middleincome countries. Curr Probl Cardiol. 2010;35(2):72-115.

5. Hatmi ZN, Tahvildari S, Gafarzadeh Motlag A, Sabouri Kashani A. Prevalence of coronary artery disease risk factors in Iran: a population based survey. BMC Cardiovasc Disord. 2007;7:32

6. Devine PJ, Carlson DW, Taylor AJ. Clinical value of carotid intimamedia thickness testing. J Nucl Cardiol. 2006;13(5):710-8.

7. Almario RU, Vonghavaravat V, Wong R, Kasim-Karakas SE. Effects of walnut consumption on plasma fatty acids and lipoproteins in combined hyperlipidemia. Am J Clin Nutr. 2001;74(1):72-9.

8. Zambon D, Sabate J, Munoz S, Campero B, Casals E, Merlos M, et al. Substituting walnuts for monounsaturated fat improves the serum lipid profile of hypercholesterolemic men and women. A randomized crossover trial. Ann Intern Med. 2000;132(7):538-46.

9. Wu L, Piotrowski K, Rau T, Waldmann E, Broedl UC, Demmelmair $\mathrm{H}$, et al. Walnut-enriched diet reduces fasting non-HDLcholesterol and apolipoprotein B in healthy Caucasian subjects: a randomized controlled cross-over clinical trial. Metabolism. 2014;63(3):382-91.

10. Kris-Etherton PM, Zhao G, Binkoski AE, Coval SM, Etherton TD. The effects of nuts on coronary heart disease risk. Nutr Rev. 2001;59(4):103-11.

11. Fukuda T, Ito H, Yoshida T. Antioxidative polyphenols from walnuts (Juglans regia L.). Phytochem. 2003;63(7):795-801.

12. Hu FB. Plant-based foods and prevention of cardiovascular disease: an overview. Am J Clin Nutr. 2003;78(3 Suppl):544S-51S.

13. Chisholm A, Mann J, Skeaff M, Frampton C, Sutherland W, Duncan A, et al. A diet rich in walnuts favourably influences plasma fatty acid profile in moderately hyperlipidaemic subjects. Eur J Clin Nutr. 1998;52(1):12-6.

14. Abbey M, Noakes M, Belling GB, Nestel PJ. Partial replacement of saturated fatty acids with almonds or walnuts lowers total plasma cholesterol and low-density-lipoprotein cholesterol. Am J Clin Nutr. 1994;59(5):995-9.

15. Sabate J, Fraser GE, Burke K, Knutsen SF, Bennett H, Lindsted KD. Effects of walnuts on serum lipid levels and blood pressure in normal men. NEngl J Med. 1993;328(9):603-7.

16. Iwamoto M, Imaizumi K, Sato M, Hirooka Y, Sakai K, Takeshita A, et al. Serum lipid profiles in Japanese women and men during consumption of walnuts. Eur J Clin Nutr. 2002;56(7):629-37.

17. Zhao G, Etherton TD, Martin KR, West SG, Gillies PJ, Kris-Etherton PM. Dietary alpha-linolenic acid reduces inflammatory and lipid cardiovascular risk factors in hypercholesterolemic men and women. J Nutr. 2004;134(11):2991-7.

18. Vega-Lopez S, Kaul N, Devaraj S, Cai RY, German B, Jialal I. Supplementation with omega3 polyunsaturated fatty acids and all-rac alpha-tocopherol alone and in combination failed to exert an anti-inflammatory effect in human volunteers:clinical and experimental. Metabolism. 2004;53(2):236-40.

19. Kris-Etherton PM, Pearson TA, Wan Y, Hargrove RL, Moriarty K, Fishell V, et al. High-monounsaturated fatty acid diets lower both plasma cholesterol and triacylglycerol concentrations. Am J Clin Nutr. 1999;70(6):1009-15.

20. Berryman CE, Grieger JA, West SG, Chen CY, Blumberg JB, Rothblat GH, et al. Acute consumption of walnuts and walnut components differentially affect postprandial lipemia, endothelial function, oxidative stress, and cholesterol efflux in humans with mild hypercholesterolemia.J Nutr. 2013;143(6):788-94.

21. Guevara I, Iwanejko J, Dembinska-Kiec A, Pankiewicz J, Wanat A, Anna P, et al. Determination of nitrite/nitrate in human biological material by the simple Griess reaction. Clin Chim Acta. 1998;274(2):177-88.

22. Danesh J, Wheeler JG, Hirschfield GM, Eda S, Eiriksdottir G, Rumley A, et al. C-reactive protein and other circulating markers of inflammation in the prediction of coronary heart disease. $N \mathrm{Engl}$ JMed. 2004;350(14):1387-97.

23. Munoz S, Merlos M, Zambon D, Rodriguez C, Sabate J, Ros E, et al. Walnut-enriched diet increases the association of LDL from hypercholesterolemic men with human HepG2 cells. J Lipid Res. 2001;42(12):2069-76.

24. Kinoshita M, Krul ES, Schonfeld G. Modification of the core lipids of low density lipoproteins produces selective alterations in the expression of apoB-100 epitopes. J Lipid Res. 1990;31(4):701-8.

25. Galeano NF, Milne R, Marcel YL, Walsh MT, Levy E, Ngu'yen TD, et al. Apoprotein B structure and receptor recognition of triglyceride-rich low density lipoprotein (LDL) is modified in small LDL but not in triglyceride-rich LDL of normal size. J Biol Chem. 1994;269(1):511-9.

26. Kasim-Karakas SE, Herrmann R, Almario R. Effects of omega-3 fatty acids on intravascular lipolysis of very-low-density lipoproteins in humans. Metabolism. 1995;44(9):1223-30.

27. Harris WS, Lu G, Rambjor GS, Walen AI, Ontko JA, Cheng Q, et al. Influence of n-3 fatty acid supplementation on the endogenous activities of plasma lipases. Am J Clin Nutr. 1997;66(2):254-60.

28. Simpoulos AP. Hearth effect of $\mathrm{w}-3$ polyunsaturated fatty acid in sea foods. World Rev NUTR Diet. 1991;66:590-2.

29. Connor WE. Alpha-linolenic acid in health and disease. Am J Clin Nutr. 1999;69(5):827-8.

30. Mantzioris E, James MJ, Gibson RA, Cleland LG. Dietary substitution with an alpha-linolenic acid-rich vegetable oil increases eicosapentaenoic acid concentrations in tissues. Am J Clin Nutr. 1994;59(6):1304-9.

31. Mantzioris E, James MJ, Gibson RA, Cleland LG. Differences exist in the relationships between dietary linoleic and alpha-linolenic acids and their respective long-chain metabolites. Am J Clin Nutr. 1995;61(2):320-4.

32. Layne KS, Goh YK, Jumpsen JA, Ryan EA, Chow P, Clandinin MT. Normal subjects consuming physiological levels of 18:3(n-3) and 20:5(n-3) from flaxseed or fish oils have characteristic differences in plasma lipid and lipoprotein fatty acid levels. J Nutr. 1996;126(9):2130-40.

33. de Deckere EA, Korver O, Verschuren PM, Katan MB. Health aspects of fish and n-3 polyunsaturated fatty acids from plant and marine origin. Eur J Clin Nutr. 1998;52(10):749-53.

34. Balk EM, Lichtenstein AH, Chung M, Kupelnick B, Chew P, Lau J. Effects of omega-3 fatty acids on serum markers of cardiovascular disease risk: a systematic review. Atherosclerosis. 2006;189(1):1930 .

35. Lavedrine F, Zmirou D, Ravel A, Balducci F, Alary J. Blood cholesterol and walnut consumption: a cross-sectional survey in France. Prev Med.1999;28(4):333-9.

36. Badimon JJ, Ibanez B. Increasing high-density lipoprotein as a therapeutic target in atherothrombotic disease. Rev Esp Cardiol. 2010;63(3):323-33.

37. Kasim SE, Stern B, Khilnani S, McLin P, Baciorowski S, Jen KL. Effects of omega-3 fish oils on lipid metabolism, glycemic control, and blood pressure in type II diabetic patients. J Clin Endocrinol Metab. 1988;67(1):1-5.

38. Parks JS, Thuren TY, Schmitt JD. Inhibition of lecithin:cholesterol acyltransferase activity by synthetic phosphatidylcholine spe- 


\section{Kalantarian $G$ et al.}

cies containing eicosapentaenoic acid or docosahexaenoic acid in the sn-2 position. J Lipid Res. 1992;33(6):879-87.

39. Kris-Etherton PM, Yu-Poth S, Sabate J, Ratcliffe HE, Zhao G, Etherton TD. Nuts and their bioactive constituents: effects on serum lipids and other factors that affect disease risk. Am J Clin Nutr. 1999;70(3 Suppl):504S-11S.

40. Feldman EB. The scientific evidence for a beneficial health relationship between walnuts and coronary heart disease. J Nutr. 2002;132(5):1062S-101S

41. Nguyen TT. The cholesterol-lowering action of plant stanol esters. J Nutr. 1999;129(12):2109-12.

42. Hallikainen MA, Sarkkinen ES, Uusitupa MI. Plant stanol esters affect serum cholesterol concentrations of hypercholesterolemic men and women in a dose-dependent manner. $J$ Nutr. 2000;130(4):767-76.
43. Dreher ML, Maher CV, Kearney P. The traditional and emerging role of nuts in healthful diets. Nutr Rev. 1996;54(8):241-5.

44. Kitts DD. Bioactive substances in food: identification and potential uses. Can J Physiol Pharmacol. 1994;72(4):423-34.

45. Anderson KJ, Teuber SS, Gobeille A, Cremin P, Waterhouse AL, Steinberg FM. Walnut polyphenolics inhibit in vitro human plasma and LDL oxidation. J Nutr. 2001;131(11):2837-42.

46. Spaccarotella KJ, Kris-Etherton PM, Stone WL, Bagshaw DM, Fishell VK, West SG, et al. The effect of walnut intake on factors related to prostate and vascular health in older men. Nutr J. 2008;7:13.

47. Burns-Whitmore B, Haddad E, Sabate J, Rajaram S. Effects of supplementing n-3 fatty acid enriched eggs and walnuts on cardiovascular disease risk markers in healthy free-living lacto-ovovegetarians: a randomized, crossover, free-living intervention study. Nutr J. 2014;13:29. 